\title{
Computer-aided tissue characterization using ultrasound-induced thermal effects: analytical formulation and in vitro animal study
}

\author{
Mohammad I. Daoud ${ }^{a}$, Parvin Mousavi $^{b}$, Farhad Imani $^{c}$, Robert Rohling ${ }^{a, d}$, \\ and Purang Abolmaesumi ${ }^{a}$ \\ ${ }^{a}$ Department of Electrical \& Computer Engineering, University of British Columbia, \\ Vancouver, BC, Canada, 'School of Computing, Queen's University, Kingston, ON, Canada, \\ ${ }^{c}$ Department of Electrical \& Computer Engineering, Queen's University, Kingston, ON, \\ Canada, ${ }^{d}$ Department of Mechanical Engineering, University of British Columbia, Vancouver, \\ BC, Canada
}

\begin{abstract}
Ultrasound radio-frequency $(\mathrm{RF})$ time series analysis provides an effective tissue characterization method to differentiate between healthy and cancerous prostate tissues. In this paper, an analytical model is presented that partially describes the variations in tissue acoustic properties that accompany ultrasound RF time series acquisition procedures. These ultrasound-induced effects, which depend on tissue mechanical and thermophysical properties, are hypothesized to be among the major contributors to the tissue typing capabilities of the RF time series analysis. The model is used to derive two tissue characterization features. The two features are used with a support vector machine classifier to characterize three animal tissue types: chicken breast, bovine liver, and bovine steak. Accuracy values as high as $90 \%$ are achieved when the proposed features are employed to differentiate these tissue types. The proposed model may provide a framework to optimize the ultrasound RF time series analysis for future clinical procedures.
\end{abstract}

Keywords: Tissue characterization, ultrasound imaging, thermal effects, acoustic propagation, time series analysis

\section{INTRODUCTION}

In the last few years, we have investigated a novel approach, named ultrasound radio-frequency (RF) time series analysis, for noninvasive tissue characterization. ${ }^{1,2}$ In this approach, an ultrasound transducer was used to acquire several hundred image frames from a specific position in the tissue, and the variations in the backscattered signals that accompany the repetitive irradiation of tissue with ultrasound were analyzed to extract tissuedependent features. These features were successfully used to characterize various animal tissues ${ }^{2}$ and differentiate between healthy and cancerous tissues in human prostate specimens. ${ }^{1}$ Despite the high accuracy of the RF time series analysis for tissue characterization, the physical processes underlying this characterization method were not understood. ${ }^{1}$

In this paper, a model is presented to study the variations in ultrasound backscattering that take place during RF time series scanning; such a model could partially explain the tissue typing capabilities of RF time series. The model is based on formulations that describe the dependence of tissue sound speed on temperature. ${ }^{3}$ Similar formulations have been used by Pereira et al. ${ }^{4}$ to develop an idealized tissue characterization procedure. In this procedure, they employed a temperature-controlled water bath to change the temperature of coronary

Further author information:

M. I. Daoud: E-mail: mdaoud@ece.ubc.ca, Telephone: 1-604-771-7489

P. Mousavi: E-mail: pmousavi@cs.queensu.ca, Telephone: 1-613-533-6000

F. Imani: E-mail: farhad@cs.queensu.ca, Telephone: 1-613-533-6000 ext 74201

R. Rohling: E-mail: rohling@ece.ubc.ca, Telephone: 1-604-822-2045

P. Abolmaesumi: E-mail: purang@ece.ubc.ca, Telephone: 1-604-827-4741

Medical Imaging 2011: Ultrasonic Imaging, Tomography, and Therapy, edited by Jan D'hooge, Marvin M. Doyley, Proc. of SPIE Vol. 7968, 79680G - ( 2011 SPIE · CCC code: 1605-7422/11/\$18 - doi: 10.1117/12.877754 
artery tissue samples and a $50 \mathrm{MHz}$ ultrasound imaging system to quantify the change in tissue sound speed as a function of temperature. Acoustic waves propagate faster in water-based tissues, such as muscle, when tissue temperature increases, while increasing tissue temperature decreases sound speed in fatty tissues. The results reported by Pereira et al. suggested that the temperature dependence of sound speed, which provides high contrast between water-based and fatty tissues, can be employed to characterize the structures of coronary artery wall based on the lipid content of each structure. This tissue characterization approach has been extended by Shi et al. ${ }^{5}$ by using a microwave source to heat a tissue-mimicking phantom composed of rubber and gelatine structures, where the temperature dependence of sound speed in the two structures is similar to water-based tissue and fatty tissue, respectively. A $10 \mathrm{MHz}$ catheter ultrasound array was used to differentiate between the rubber and gelatine structures based on the variation in sound speed as a function of temperature. In a recent study, Kim et al. ${ }^{6}$ employed a therapeutic ultrasound array as a heat source to raise the temperature of tissue samples and used a $10 \mathrm{MHz}$ diagnostic ultrasound array, placed in a plane perpendicular to the heat source, to differentiate fatty and water-based tissues based on the temperature dependence of sound speed.

The mathematical formulations presented in this paper are focused on employing an ultrasound array operating at clinical frequency $(7.2 \mathrm{MHz})$ to both raise tissue temperature and measure the associated change in tissue sound speed. This scanning set-up is similar to the scanning procedure of the RF time series characterization method. A new characterization method is also introduced that uses two features for tissue classification that estimate the ultrasound-induced thermal change in tissue sound speed based on ultrasound backscattered signals. The validity of the method for tissue classification is demonstrated by differentiating three water-based animal tissues. Classifying water-based tissues is more challenging than the problem of differentiating water-based tissue from fatty tissues. The mathematical formulations described in this paper provide a framework to study the RF time series analysis and optimize its performance for clinical procedures.

\section{METHODS}

\subsection{Theory}

Acoustic propagation in tissue changes tissue temperature as expressed in Equation (1): ${ }^{7,8}$

$$
\dot{T}=\kappa \nabla^{2} T-\frac{T}{\tau}+\frac{q_{v}}{c_{v}}
$$

where $T$ is tissue temperature, $\dot{T}$ is the rate of tissue temperature change, $\kappa$ is tissue thermal diffusivity, $\tau$ is the perfusion time constant, $q_{v}$ is the rate of ultrasound-induced heat production per unit volume, and $c_{v}$ is the heat capacity per unit volume. The ultrasound-induced heat source can be written as: ${ }^{9}$

$$
q_{v}=2 \alpha I
$$

In Equation (2), $I$ is the ultrasound intensity and $\alpha$ is the absorption coefficient.

The ultrasound-induced change in tissue temperature changes tissue sound speed and expands tissue volume. The rate of thermal expansion in soft tissue is much smaller than the rate of sound speed change as a function of tissue temperature. ${ }^{3}$ Therefore, the effect of thermal expansion can be ignored to simplify the analysis. The ultrasound-induced change in tissue sound speed alters two physical quantities that affect ultrasound backscattering: the transmit-receive round trip time of echoes backscattered from tissue and the temporal spacing between tissue scatterers. The effect of ultrasound propagation and the associated tissue temperature increase on both physical quantities are analyzed in this section. The analysis will be limited to the axial direction, $z$, to reduced its complexity.

When the transducer is placed at $z=0$, the round trip time, $t(a)$, required to transmit a pulse to a point located at $z=a$, and receive an echo from the same point is given by: ${ }^{3}$

$$
t(a)=2 \int_{0}^{a} \frac{1}{c(T, z)} d z
$$

where $c(T, z)$ is the temperature-dependent sound speed as a function of the axial coordinate, $z$. Using Equation (3), it can be shown that there is a time shift, $\delta(T, a)$, between the signal received from a point located at 
$z=a$ after acoustic exposure (which changes tissue temperature and sound speed) and the signal received from the same point before exposure. The time $\operatorname{shift} \delta(T, a)$ can be written as: ${ }^{3}$

$$
\delta(T, a)=2 \int_{0}^{a}\left[\frac{1}{c(T, z)}-\frac{1}{c_{0}(T, z)}\right] d z
$$

In Equation $(4), c_{0}(T, z)$ is the spatially-dependent tissue sound speed before acoustic exposure and $c(T, z)$ is the tissue sound speed after the exposure. A negative time shift value indicates that the ultrasound-induced rise in temperature increases tissue sound speed, and hence reduces the transmit-receive round trip time of ultrasound signals. On the other hand, a positive time shift value implies that ultrasound heating of tissue decreases tissue sound speed. Positive time shift values are usually observed in fatty tissues, and negative time shift values take place in water-based tissues. ${ }^{4,6}$

The ultrasound-induced change in tissue temperature changes the temporal spacing between scatterers as well. For example, increasing the temperature in water-based tissue, such as muscle, increases tissue sound speed, and hence the propagation time between tissue scatterers (or the temporal spacing between scatterers) becomes lower. The amount of change in the temporal spacing between scatterers is determined by the tissuedependent coefficient that describes the sound speed variation as a function of tissue temperature. For fatty tissue, the ultrasound-induced rise in tissue temperature decreases tissue sound speed, and hence increases the temporal spacing between scatterers. Since the spatial arrangement of scatterers within tissue is an important determinant of ultrasound backscattering, ${ }^{10}$ the ultrasound-induced change in tissue sound speed alters the characteristics of the backscattered ultrasound signals, including the signal shape. It is worth mentioning that the change in the temporal spacing between scatterers as a function of tissue temperature has been used by Seip et $a .^{11}$ to monitor tissue temperature.

Acoustic propagation in tissue changes the temperature, with temperature described in Equations (1) and (2), and this thermal effect varies from one tissue to another depending on tissue mechanical and thermophysical properties. The change in temperature alters tissue sound speed, which in turn introduces a phase shift in the backscattered signals as shown in Equation (4). Moreover, the ultrasound-induced variation in tissue temperature and sound speed changes the temporal spacing between scatterers, which in turn alters the shape of the backscattered signals. The change in tissue temperature and sound speed due to acoustic exposure can be estimated by measuring the phase shift and shape variations of the ultrasound backscattered signals received after acoustic exposure compared to signals received before the exposure. The measured ultrasound-induced changes in ultrasound backscattering, which depend on tissue properties, can be used for tissue characterization.

\subsection{Experimental Ultrasound Imaging}

Ultrasound imaging is performed using a Sonix MDP system (Ultrasonix Inc., Richmond, British Columbia, Canada). The scanner employs a L14-5/38 linear array transducer that has 128 piezoelectric elements and a center frequency of $7.2 \mathrm{MHz}$. At each scan acquisition, $1500 \mathrm{RF}$ frames are acquired with a rate of 500 frames per second. Each frame is composed of 20 scan lines with a lateral spacing of $0.3 \mathrm{~mm}$. An individual scan line is created using 100 transducer elements configured to produce a focal distance of $20 \mathrm{~mm}$ and an $f$-number of 0.67. A long transmitted pulse composed of six cycles is used in the acquisition. The received RF signals are sampled using an analog to digital converter with a sampling frequency of $40 \mathrm{MHz}$.

Two chicken breast specimens, two bovine liver specimens, and two bovine steak specimens are scanned using the linear array. All specimens are fresh store-bought tissues. Each specimen is scanned at two independent locations that are spaced along the lateral axis by a distance greater than the lateral dimension of the transducer. Five 1500-frame sequences are acquired at each location in the specimen, with a seven-minute waiting time between two successive sequences. The waiting time is required to cool the tissue, and hence reduce the effect of the residual temperature created by previous RF sequence acquisitions. A schematic representation of the scanning set-up is shown in Figure 1. In this set-up, the tissue is placed on a tissue-mimicking phantom to reduced acoustic reflection from the lower surface of the tissue. A standoff pad of thickness $10 \mathrm{~mm}$ is placed between the transducer surface and the tissue to remove the possible influence of heat created by the temperature rise of the piezoelectric elements. 


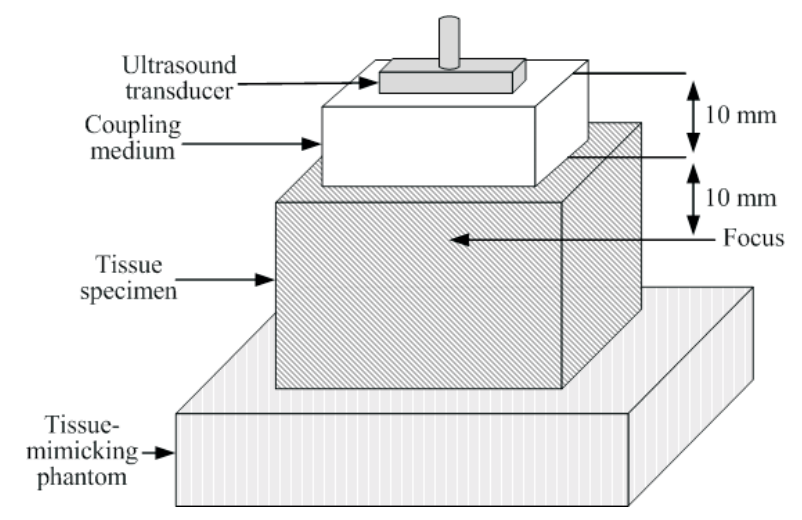

Figure 1. Ultrasound imaging set-up.

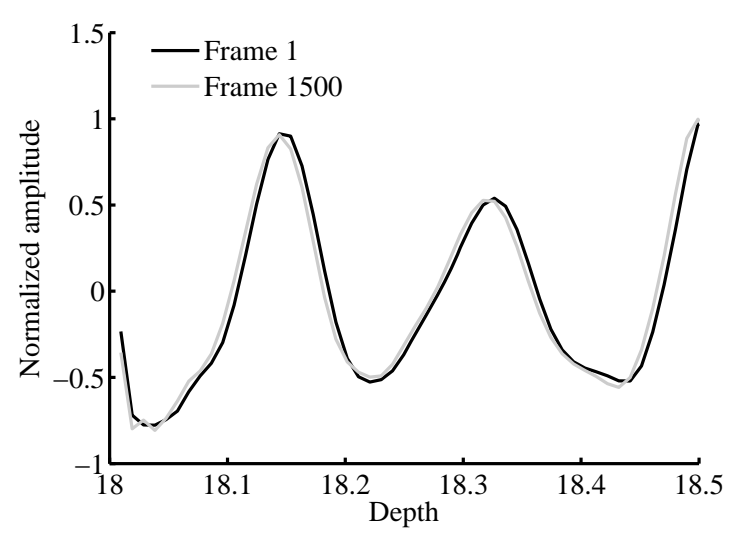

(a)

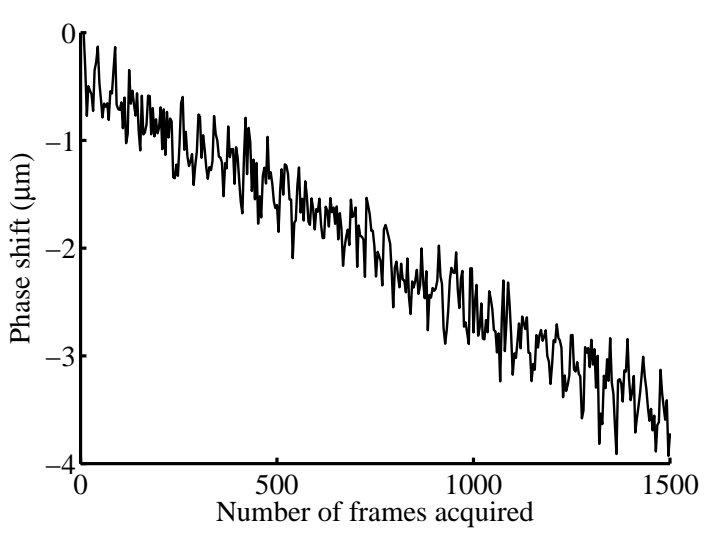

(b)

Figure 2. (a) Two RF signals acquired from a chicken breast specimen at frame 1 and frame 1500 of a sequence acquisition, and (b) the phase shift between the backscattered signals received from an ROI in a chicken breast specimen after acoustic exposure with respect to the matching signal received before exposure as a function of number of frames acquired.

\subsection{Feature Extraction}

As mentioned before, the ultrasound-induced change in tissue sound speed creates a phase shift between the RF signals received at different instances of an RF sequence acquisition. For example, Figure 2a shows the signal received from a region of interest (ROI) in a chicken breast specimen at the beginning of a sequence acquisition, i.e. obtained from frame 1, and the matching signal received from frame 1500, when both signals are processed to compensate for the phase shift introduced in the standoff pad. The two signals exhibit a phase shift of 4 $\mu \mathrm{m}$, which represents the ultrasound-induced phase shift in the chicken breast tissue. Since tissue temperature increases as new frames are acquired, the shift between an RF signal in frame $i$ and the matching signal in frame 1, which we call the reference frame, is correlated with $i$. The monotonic increase in phase shift as a function of number of frames acquired is shown in Figure 2b. That figure presents the phase shift between signals received from an ROI in a chicken breast specimen as the RF sequence is acquired and the matching signal in the reference frame, after compensating for the phase shift introduced in the standoff pad. The phase shifts in the backscattered signals due to acoustic propagation that are shown in Figures 2a and 2b agree with Equation (4). The ultrasound-induced phase shift as a function of number of frames acquired is estimated for the two locations scanned in each specimen at four ROIs spaced from the transducer by distances of 19.50, 19.75, 20.00, and $20.25 \mathrm{~mm}$ along the axial dimension. The motion estimation algorithm reported in reference 12 is used to compute the phase shifts. The effect of the ultrasound-induced phase shift in the standoff pad is eliminated by compensating for the phase shift observed in the tissue region near the tissue-standoff pad interface. 
Table 1. Two-way classification accuracies between bovine steak, bovine liver, and chicken breast obtained when the support vector machine classifier employs the ultrasound-induced phase shift feature, the ultrasound-induced variation in the signal's shape feature, and both the phase shift and variation in shape features.

\begin{tabular}{cccc}
\hline Tissue types & Phase shift feature & Shape variation feature & $\begin{array}{c}\text { Phase shift and } \\
\text { shape variation features }\end{array}$ \\
\hline Bovine steak and chicken breast & $87.7 \%$ & $64.5 \%$ & $90.6 \%$ \\
Bovine steak and bovine liver & $81.4 \%$ & $69.5 \%$ & $90.2 \%$ \\
Bovine liver and chicken breast & $72.8 \%$ & $67.6 \%$ & $79.5 \%$ \\
\hline
\end{tabular}

The variation in tissue sound speed during a sequence acquisition alters the temporal spacing between tissue scatterers, as discussed in Section 2.1. The change in the temporal spacing between tissue scatterers varies the shape of the backscattered signals, as can be depicted around the positive and negative peaks of the RF signals shown in Figure 2a. The change in the signal's shape is quantified for the two locations scanned in each tissue specimen at the four ROIs described above by computing the correlation coefficient between the signals received from the ROI as the RF sequence is acquired and the matching signal in the reference frame.

Two features are computed for each ROI that separately quantify the variations in the phase and the shape of the backscattered signals. The first feature, which describes the change in phase, is calculated by fitting a line to the ultrasound-induced phase shifts computed for the ROI as a function of number of frames acquired, and then computing the slope of the fitted line. The second feature, which describes the change in the signal's shape, is computed by fitting a line to the correlation coefficients computed for the ROI as a function of number of frames acquired, and then calculating the norm of the differences between the experimental correlation coefficients and the matching values in the fitted line.

\subsection{Tissue Classification}

A support vector machine classifier ${ }^{13}$ is used to classify the ROIs. A tenfold cross validation procedure is employed, in which the first $90 \%$ of the features set is used to train the classifier and the remaining $10 \%$ is used for validation. To avoid over-fitting the classifier, the features extracted for the ROIs are sorted randomly, and then the tenfold classification procedure is run. The process of randomly sorting the features and running the classifier is repeated for 50 trials, and the mean value of the classification accuracies obtained for the 50 train-validate trials is reported.

\section{RESULTS}

The two-way classification accuracies between each pair of the three tissue types examined are shown in Table 1 when the classifier employs the phase shift feature separately, the variation in the signal's shape feature separately, and both the phase shift and variation in shape features. Accuracies greater than $90 \%$ are obtained when the classifier combined the two features to differentiate the bovine steak from the two other tissue types. However, when the bovine liver and chicken breast tissues are classified, the accuracy achieved using the combined phase shift and shape variation features is around $80 \%$. The high classification accuracy between bovine steak and the two other tissues might be attributed to the high water content in chicken breast and bovine liver compared to bovine steak. The classification accuracies achieved using the phase shift feature are lower than the accuracies obtained by combing the two features. However, the classification accuracies of the phase shift feature are higher than the accuracy values of the shape variation feature.

\section{CONCLUSION}

This paper provides an analytical model and experiments to partially explain the tissue characterization properties of RF time series. We use the temperature dependence of tissue sound speed to characterize tissue by employing a conventional ultrasound array to both heat tissue and quantify the associated change in sound speed. Two features, namely the changes in the phase and the shape of the backscattered ultrasound signals, are employed to classify tissue based on the ultrasound-induced variation in tissue sound speed. The performance 
of the two features is validated by classifying three water-based tissue types: chicken breast, bovine steak, and bovine liver. Classification accuracies greater than $90 \%$ are achieved when bovine steak is differentiated from chicken breast and bovine liver. Moreover, classification accuracy of $79.5 \%$ is obtained when chicken breast is differentiated from bovine liver.

\section{ACKNOWLEDGMENTS}

The authors thank Dr. Reza Zahiri-Azar and Prof. Tim Salcudean for providing the source code of the motion estimation algorithm of reference 12. Samuel Frew and Prof. Tim Salcudean are acknowledged for constructive conversations about the ultrasound-induced thermal effects in tissues. This research is supported by the Natural Sciences and Engineering Research Council of Canada (NSERC) and the Canadian Institutes of Health Research (CIHR). Mohammad I. Daoud holds an NSERC Postdoctoral Fellowship.

\section{REFERENCES}

[1] Moradi, M., Abolmaesumi, P., Siemens, D., Sauerbrei, E., Boag, A., and Mousavi, P., "Augmenting detection of prostate cancer in transrectal ultrasound images using SVM and RF time series," IEEE Trans. Biomed. Eng. 56, 2214-2224 (2008).

[2] Moradi, M., Mousavi, P., Isotalo, P., Siemens, D., Sauerbrei, E., and Abolmaesumi, P., "A new approach to analysis of RF ultrasound echo signals for tissue characterization: animal studies," in Proc. SPIE 6513, $65130 \mathrm{P}-1-65130 \mathrm{P}-10$ (2007).

[3] Maass-Moreno, R. and Damianou, C., "Noninvasive temperature estimation in tissue via ultrasound echoshifts. Part I. Analytical model," J. Acoust. Soc. Am. 100, 2514-2521 (1996).

[4] Pereira, F., Machado, J., and Foster, F., "Ultrasound characterization of coronary artery wall in vitro using temperature-dependent wave speed," IEEE Trans. Ultrason. Ferroelectr. Freq. Control. 50, 14741485 (2003).

[5] Shi, Y., Witte, R., and O'Donnell, M., "Identification of vulnerable atherosclerotic plaque using IVUS-based thermal strain imaging," IEEE Trans. Ultrason. Ferroelectr. Freq. Control. 52, 844-850 (2005).

[6] Kim, K., Huang, S.-W., Hall, T., Witte, R., Chenevert, T., and O'Donnell, M., "Arterial vulnerable plaque characterization using ultrasound-induced thermal strain imaging (TSI)," IEEE Trans. Biomed. Eng. 55, 171-180 (2008).

[7] Nyborg, W., "Solutions of the bio-heat transfer equation," Phys. Med. Biol. 33, 785-792 (1988).

[8] Pennes, H., "Analysis of tissue and arterial blood temperatures in the resting human forearm," J. Appl. Physiol. 1, 93-122 (1948).

[9] Palmeri, M. and Nightingale, K., "On the thermal effects associated with radiation force imaging of soft tissue," IEEE Trans. Ultrason. Ferroelectr. Freq. Control. 51, 551-565 (2004).

[10] Hunt, J., Worthington, A., and Kerr, A., "The subtleties of ultrasound images of an ensemble of cells: simulation from regular and more random distributions of scatterers," Ultrasound Med. Biol. 21, 329-341 (1995).

[11] Seip, R. and Ebbini, E., "Noninvasive estimation of tissue temperature response to heating fields using diagnostic ultrasound," IEEE Trans. Biomed. Eng. 42, 828-839 (1995).

[12] Zahiri-Azar, R. and Salcudean, S., "Motion estimation in ultrasound images using time domain cross correlation with prior estimates," IEEE Trans. Biomed. Eng. 53, 1990-2000 (2006).

[13] Chang, C.-C. and Lin, C.-J., "Libsvm: a library for support vector machines," http://www.csie.ntu.edu.tw/ cjlin/libsvm (2001). 